\title{
Overexpanding coronal mass ejections at high heliographic latitudes: Observations and simulations
}

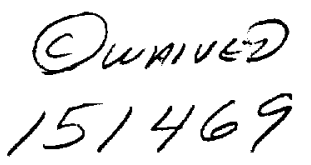

\author{
J. T. Gosling, Pete Riley, and D. J. McComas \\ Los Alamos National Laboratory, Los Alamos, New Mexico
}

\author{
V. J. Pizzo \\ Space Environment Center, National Oceanic and Atmospheric Administration, Boulder, Colondo
}

\begin{abstract}
Ulysses observations reveal that most coronal mass ejections (CMEs) observed in the solar wind far from the Sun at high heliographic latitudes have large radial widths and are still expanding as they pass the spacecraft. CME radial widths ranging between 0.5 and $2.5 \mathrm{AU}$ have been observed at heliocentric distances between 1.4 and $4.6 \mathrm{AU}$ and at latitudes greater than $22^{\circ}$. A CME may expand simply because it is ejected from the Sun with a leading edge speed that is greater than its trailing edge speed. Rarefaction waves produced by relative motion between a $\mathrm{CME}$ and the surrounding wind also can cause a CME to expand. Finally, a CME may expand because it is ejected into the wind with an internal pressure that is greater than that of the surrounding wind. In the latter case, which we have called "overexpansion," the expansion tends to drive compressive waves into the surrounding solar wind; these waves commonly steepen into shocks at large distances from the Sun. The relative importance of these various expansion processes differs from event to event depending upon initial conditions within the CME and the surrounding wind. Using Ulysses observations and a simple one-dimensional, gasdynamic code, we have explored how initial conditions affect the radial evolution of solar wind disturbances associated with overexpanding CMEs. We find good qualitative agreement between the results of our simulations and Ulysses observations of such disturbances.
\end{abstract}

\section{Introduction}

Coronal mass ejections (CMEs) are transient events in which large amounts of material from the solar atmosphere are ejected into the solar wind (see, for example, Hundhausen [1988]: and Kahler [1988]). They originate in closed magnetic field regions in the corona where the magnetic field normally is sufficiently strong to constrain the plasma from expanding outward. CMEs play a central role in the long-term evolution of the corona [e.g., Hundhausen, 1997] and are the prime link between solar activity, large transient solar wind disturbances, and large geomagnetic storms [e.g., Gosling. 19931.

Close to the solar surface CMEs typically have radial extents of less than a solar radius, but they commonly expand radially as they propagate away from the Sun. As distinguished by counterstreaming suprathermal electron fluxes [Gosling et al., 1987], the average CME (i.e., the material ejected in the solar event) has a radial width at Earth's orbit, one astronomical unit ( $1 \mathrm{AU}$ ) from the Sun, of $-0.2 \mathrm{AU}$, although the range of observed CME widths is substantial. Most CMEs are still expanding as they pass $1 \mathrm{AU}$ since their leading edge speeds typically (but not always) exceed their trailing edge speeds. Consequently, CMEs in the outer heliosphere often have large radial extents.

CME expansions in the solar wind can result from any of several processes. A CME may expand simply because it is ejected from the Sun with a leading edge speed that exceeds its

Copyright 1998 by the American Geophysical Union.

Paper number $97 J A 01304$.

0148-0227/98/97JA-01304\$09.00 rrailing edge speed. Another possibility is that expansion is a CME's dynamic response to a rarefaction wave produced by its motion relative to the surrounding solar wind [e.g.. Gosling and Riley, 1996]. Finally, a CME may expand because it is ejected into the solar wind with an internal pressure that exceeds that of the surrounding ambient plasma [Gosling, 1976; Burlaga et al., 1981; Klein and Burlaga, 1982; Chen, 1996]. We have used the term "overexpansion" to describe CME events where the expansion is driven by such a high initial internal pressure [Gosling et al., 1994a]. The higher internal pressure can be a result of a higher density, a higher temperature, a higher magnetic field strength, or some combination thereof. The relative importance of these various expansion processes differs from event to event depending on initial conditions in the surrounding solar wind and the physical character of the CME.

Ulysses, whose payload includes a Los Alamos solar wind plasma experiment [Bame et al., 1992], was launched on October 6, 1990, and is now in an orbit that has carried the spacecraft nearly over both poles of the Sun. We have previously reported a new class of forward-reverse shock pair events discovered in the Ulysses observations obtained at high heliographic latitudes [Gosling et al., 1994c]. These events are caused by the overexpansion of CMEs that have speeds comparable to that of the surrounding solar wind plasma. Of six certain CMEs observed poleward of $531^{\circ}$ during Ulysses' initial transit to high southern latitudes, three had associated shock pairs of this nature. We have shown that many of the essential aspects of these CME/shock pair events can be understood with the help of simulations using a simple, one-dimensional, adiabatic (except at shocks), gasdynamic code [Hundhausen and Gentry, 1969]. This code predicts too 
strong an interaction between newly ejected solar material and the ambient solar wind because it neglects azimuthal and meridional motions of the plasma that help relieve pressure stresses. Moreover, magnetic forces are not explicitly included. Despite these limitations, such calculations provide a starting point for understanding the Ulysses high-latitude observations. Excellent descriptions of the use of this and other codes for modeling coronal and solar wind disturbances can be found in reviews by Hundhausen [1985], Pizzo [1985]. and Steinolfson [1985].

An expanding, high-pressure CME will produce leading and trailing shocks only under certain conditions. For example. if the CME speed is considerably greater than that of the ambient wind ahead, as is often the case at low heliographic latitudes, the evolution of the disturbance associated with the CME is dominated by pressure gradients induced by the relative motion rather than by over-expansion. Indeed, simultaneous high- and low-latitude observations by Ulysses and IMP 8 of a CME-driven disturbance in February and March 1994 indicate that a fast CME having a high internal pressure can produce dramatically different disturbances at high and low latitudes owing to latitudinal gradients in the ambient wind [Gosling et al., 1995a]. Our one-dimensional simulations have provided a reasonable explanation for most of the essential differences observed at high and low latitudes in the February/March 1994 disturbance, although a multidimensional simulation is required to gain more complete understanding of the global evolution of such a disturbance [Riley et al., 1997].

Our purpose here is to report and discuss Ulysses observations of overexpanding CMEs at high heliographic latitudes with emphasis on those events that did not produce forward-reverse shock pairs at the spacecraft. We use simple one-dimensional. gasdynamic simulations to illustrate how different initial conditions in the ambient wind and different physical characteristics of the CMEs produce different disturbance signatures far from the Sun. In particular, we show how reasonable choices of initial conditions and boundary conditions can produce heliospheric events similar to those observed by Ulysses far from the Sun at high heliographic latitudes. We have used our experience with the simulations to choose initial perturbations close to the Sun that we believed would produce disturbances similar to what we observe in the Ulysses events. However, we have not attempted to reproduce all details of the observed Ulysses events with our numerical simulations. Indeed, the limitations of one-dimensional, gasdynamic calculations and our lack of knowledge of the proper boundary conditions close to the Sun effectively prohibit that from occurring. Nevertheless, our simulations have produced disturbance profiles far from the Sun that are qualitatively, and sometimes quantitatively, similar to those observed. We thus believe they provide a good starting point for obtaining an understanding of the dynamics and radial evolution of CMEs ejected into the high-speed solar wind at high heliographic latitudes.

\section{Observations and Simulations}

As already noted, six certain CMEs (i.e., their interplanetary counterparts) were detected by the plasma experiment on Ulysses poleward of S33" during Ulysses' initial transit to high southern latitudes in 1993 and 1994. These CMEs were distinguished in the plasma data primarily by the counterstreaming (along the magnetic field) suprathermal electron signature characteristic of closed magnetic field lines in the solar wind [e.g., Gosling, 1990, 1996]; however, all of these CMEs also had distinct magnetic field signatures as well. Since then, two additional CMEs have been detected at relatively high solar latitudes. The first of these was encountered in February 1995 at $\$ 22^{\circ}$ when the spacecraft was at the southern edge of the low-latitude band of solar wind variability at a heliocentric distance of $\sim 1.4 \mathrm{AU}$ [Gosling et al., 1995b]. The second was encountered in October 1996 at $\mathrm{N} 24^{*}$ when Ulysses was close to the northern edge of the band of variability at $4.5 \mathrm{AU}$ [Gosling et al., 1997]. Various aspects of the eight certain high-latitude CME events observed by Ulysses are summarized in Table 1.

Most of the high-latitude CMEs observed by Ulysses had higher front edge speeds than back edge speeds and thus were expanding as they passed over the spacecraft. This expansion usually produced relatively wide CMEs with low internal plasma densities and pressures. Figure I shows a plot of 1 hour averages of solar wind speed, proton density, and proton thermal pressure measured by the Ulysses plasma experiment during a 6-month interval in 1993 as Ulysses moved to ever higher southern solar latitudes in the middle of 1993. Three of the high-latitude CMEs observed by Ulysses occurred in this interval. All three occurred on declining speed gradients and all are distinguished in Figure I by abnormally low proton densities and pressures. Despite the evidence for expansion in these events, only the event in early June produced a forwardreverse shock pair.

\section{April 1994 Event}

Figure 2a shows selected plasma parameters for an overexpansion event observed in April 1994 when Ulysses was at $3.2 \mathrm{AU}$ and $\mathrm{S} 61^{\circ}$. This event was associated with a large solar soft X ray event observed by Yohkoh on April 14 1994 [e.g.. Alexander et al., 1994; McAllister et al., 1996; Weiss et al., 1996]. The CME material was identified in the plasma data by the presence of counterstreaming halo electrons. indicating that much of the CME was threaded by field lines attached to the Sun at both ends [e.g., Gosling, 1996]. The overall speed of the CME was comparable to that of the ambient wind ahead and behind; however, the speed declined from the front to the rear of the event, indicating that the CME was still expanding as it passed over Ulysses. Minima in proton density and proton pressure occurred near the center of the CME, while maxima in these quantities occurred immediately downstream from the relatively weak shocks. which stood off from the outer edges of the CME. The shocks in this and other examples have been identified using the combined plasma and magnetic fjeld data from Ulysses. Note that the reverse shock stood off more from the CME than did the forward shock. We calculate that the CME at Ulysses had a radial width of $-0.46 \mathrm{AU}$, while the total disturbance width from forward to reverse shock was $-1.3 \mathrm{AU}$.

We have performed a one-dimensional, gasdynamic simulation of the April 1994 event using a versatile numerical code (ZEUS) [Stone and Norman, 1992] that we have benchtested favorably against the older Hundhausen and Gentry code. Our present calculations extend from an inner boundary at $0.14 \mathrm{AU}$, which lies well outside the critical point where the solar wind goes supersonic, to an outer boundary at $6.0 \mathrm{AU}$. By starting the simulations outside the critical point we avoid questions associated with the initiation and initial 
Table 1. Ulysses High-Latitude Coronal Mass Ejection Events

\begin{tabular}{|c|c|c|c|c|c|c|c|c|}
\hline Start & End & $\begin{array}{c}\text { CME } \\
\text { Width, } \\
\text { AU }\end{array}$ & $\begin{array}{l}\mathrm{CME} \\
\text { Center } \\
\text { Speed, } \\
\mathrm{km} / \mathrm{s} \\
\end{array}$ & $\begin{array}{c}\text { Ambient } \\
\text { Leading } \\
\text { Speed, } \\
\mathrm{km} / \mathrm{s}\end{array}$ & $\begin{array}{c}\text { Ambient } \\
\text { Trailing } \\
\text { Speed, } \\
\mathrm{km} / \mathrm{s} \\
\end{array}$ & $\begin{array}{c}\text { Associated } \\
\text { Shocks* }\end{array}$ & $\begin{array}{r}\text { Heliocentric } \\
\text { Distance. AU } \\
\end{array}$ & $\begin{array}{c}\text { Latitude, } \\
\text { deg }\end{array}$ \\
\hline $\begin{array}{c}\text { June } 9,1993 \\
2130\end{array}$ & $\begin{array}{c}\text { June } 13,1993 \\
0130\end{array}$ & 1.2 & 740 & 800 & 675 & $\begin{array}{l}\text { F June } 9 \\
0130 \\
\text { R June } 14 \\
0415\end{array}$ & 4.6 & S 33 \\
\hline $\begin{array}{c}\text { July } 20,1993 \\
0115\end{array}$ & $\begin{array}{c}\text { July } 25,1993 \\
1130\end{array}$ & 2.0 & 610 & 750 & 710 & $\begin{array}{l}\text { R July } 26 \\
1000\end{array}$ & 4.5 & S 35 \\
\hline $\begin{array}{c}\text { Aug. } 27,1993 \\
2000\end{array}$ & $\begin{array}{c}\text { Aug. } 30,1993 \\
1100\end{array}$ & 1.2 & 750 & 800 & 700 & $\begin{array}{l}\text { R Sept. } 1 \\
0655\end{array}$ & 4.4 & S 38 \\
\hline $\begin{array}{c}\text { Feb. } 9,1994 \\
1400\end{array}$ & $?$ & $?$ & 750 & 780 & ? & - & 3.6 & S 52 \\
\hline $\begin{array}{c}\text { Feb. } 27,1994 \\
1030\end{array}$ & $\begin{array}{c}\text { Feb. } 28,1994 \\
1800\end{array}$ & 0.5 & 760 & 740 & 770 & $\begin{array}{l}\text { F Feb. } 26 \\
131.0 \\
\text { R March 1 } \\
1550\end{array}$ & 3.5 & S 54 \\
\hline $\begin{array}{c}\text { April } 21,1994 \\
0530\end{array}$ & $\begin{array}{c}\text { April } 22,1994 \\
0800\end{array}$ & 0.5 & 740 & 750 & 730 & $\begin{array}{l}\text { F April } 20 \\
0900 \\
\text { R April } 23 \\
1045\end{array}$ & 3.2 & S 61 \\
\hline $\begin{array}{c}\text { Feb. 3, } 1995 \\
0730\end{array}$ & ? & $?$ & 690 & 750 & 570 & $\begin{array}{l}\text { F Feb. } 3 \\
0310\end{array}$ & 1.4 & S 22 \\
\hline $\begin{array}{c}\text { Oct. } 14,1996 \\
0830\end{array}$ & $\begin{array}{c}\text { Oct. } 21,1996 \\
1530\end{array}$ & 2.5 & 630 & 730 & 550 & $\begin{array}{l}\text { F Oct. } 13 \\
2150\end{array}$ & 4.4 & N 24 \\
\hline
\end{tabular}

* $F$ and $R$ refer to forward and reverse shocks, respectively.

acceleration of the CMEs. Speed, density, and temperature are first held steady at the inner boundary until a stationary, highly supersonic flow with a speed of $750 \mathrm{~km} \mathrm{~s}^{-1}$ at $6.0 \mathrm{AU}$ and a density of $2.5 \mathrm{~cm}^{-3}$ at $1 \mathrm{AU}$, matching average highlatitude flow conditions observed by Ulysses [Phillips et al., 1994], fills the computational mesh. In order to simplify comparisons between various simulation runs using the Zeus code, these same initial conditions are employed throughout this paper even though the ambient wind ahead of the observed high-latitude CMEs varies somewhat from event to event.

Figure $2 b$ shows the temporal profile of a simulated disturbance at 3.2 AU (Ulysses' heliocentric distance at the time of the April 1994 event) initiated at the inner boundary by increasing the density by a factor of four in a bell-shaped pulse 10-hours wide while simultaneously holding the temperature and speed constant there. This mimics the ejection of a dense CME from the Sun whose initial radial width is $0.17 \mathrm{AU}$, whose internal pressure is higher than that of the surrounding solar wind, and whose speed is the same as that of the ambient wind. Figure 3 shows the radial evolution of the disturbance 55 and 194 hours after initiation, indicates the initial perturbations for the simulation, and documents the expansion of the CME.

Owing to its initial high internal pressure, the CME expands as it travels out from the Sun so that at 3.2 AU it has a width of $0.40 \mathrm{AU}$. The overall disturbance width at $3.2 \mathrm{AU}$ is $-0.67 \mathrm{AU}$ since the expansion drives a forward wave into the ambient wind ahead and a reverse wave into the trailing wind. These pressure waves steepen into shocks by the time they reach the spacecraft position, with maxima in density, temperature, and pressure occurring immediately downstream from the shocks. Ambient wind encountering the forward wave is accelerated, while ambient wind encountering the reverse wave is decelerated. The expansion also produces a declining speed gradient across the CME and causes the density, temperature (not shown), and pressure within the CME far from the Sun to be lower than that in the ambient wind immediately surrounding the disturbance. The rate of expansion of the CME slows with increasing heliocentric distance as the CME interacts with the surrounding ambient solar wind; however, the CME continues to expand to the outer edge of the simulation at $6 \mathrm{AU}$.

Comparison between the observed disturbance shown in Figure 2a and the simulated disturbance shown in Figure $2 \mathbf{b}$ reveals both the strengths and weaknesses of the simulation. Overall, the simulated disturbance profile bears a marked resemblance to that observed by Ulysses in April 1994. In particular, the simulation produces a disturbance that is slightly asymmetrical about the center of a slowly expanding $\mathrm{CME}$, with relatively weak shocks propagating forward and backward into the surrounding solar wind plasma, with a pressure minimum near the center of the CME, and pressure maxima immediately downstream of the shocks, as in the observed event. The reverse shock in the simulation also stands off from the back edge of the CME more than the forward shock does from the front edge, as in the observations. This and other small asymmetries in the simulated disturbance are a consequence of the continued evolution (expansion) of the disturbance as it passes over a 


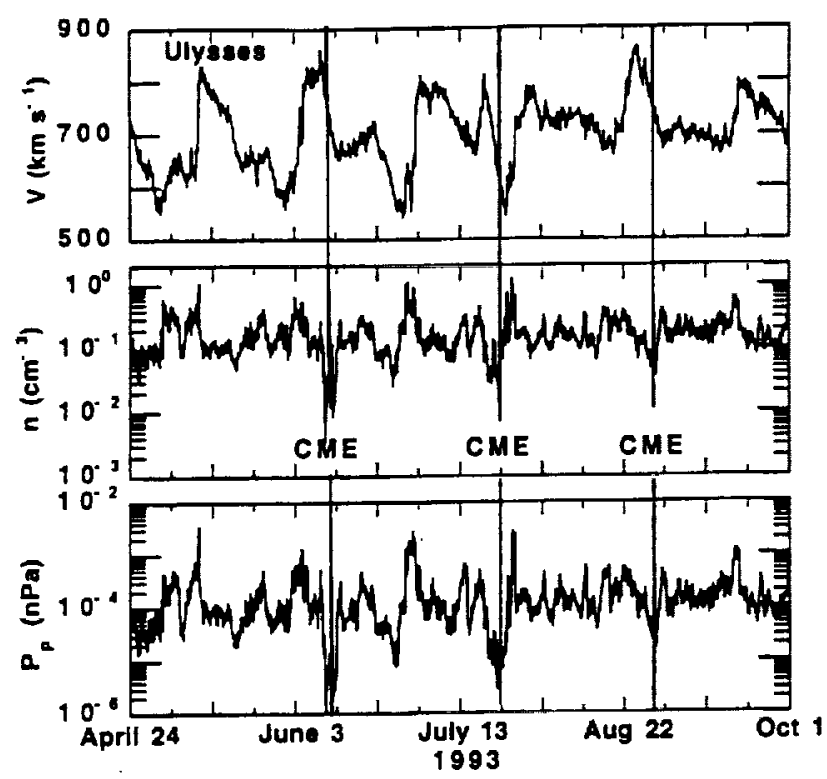

Figure 1. Ulysses measurements of solar wind speed, proton density, and proton pressure for a 160-day interval in 1993 as the spacecraft moved to ever higher southern solar latitudes. Vertical lines mark the centers of three acoronal mass ejections (CMEs) detected in this interval. All of the CMEs are characterized by high, but declining, solar wind speed and low density and pressure.

fixed point in space. The simulation also produces a CME width comparable to that observed.

On the other hand, the edge-center-edge contrast in density and pressure within the CME is considerably greater in the simulation than in the observed event, and the shocks in the simulation do not stand off at as great a distance from the edges of the CME as they do in the observed event, presumably because the characteristic speed with which pressure disturbances propagate in the simulation is less than in the real solar wind. Moreover, the absolute gas pressure at 3.2 AU in the simulation is almost an order of magnitude less than was observed; this difference is a consequence of using an adiabatic treatment, which causes temperature to fall off more rapidly with distance than is observed, and our decision to choose the initial state in the simulation to match observed speeds and densities, rather than observed pressures, in the ambient solar wind at high heliographic latitudes. Thus the discrepancies between observations and simulations can probably be ascribed to limitations inherent in our use of onedimensional, adiabatic, gasdynamic calculations and to differences between our chosen initial conditions. both within the ambient wind and within the CME. and those within the real solar wind. For example. although we find it convenient to mimic an initial high internal CME pressure with a density pulse, in real events high internal pressure may also be a consequence of either high temperature or high magnetic field strength or both Despite differences between the observed and simulated disturbances it is clear that (1) the April 1994 Ulysses event was produced by overexpansion of a CME traveling at about the same speed as the surrounding solar wind, and (2) most of the essential aspects of the disturbance evolution are reproduced by the simulation. This simulation also does a reasonably good job of reproducing the essential observational aspects of the late February 1994 event. We note that the introduction of a sizable initial speed gradient across the CME in the simulation would produce a disturbance in considerably poorer agreement with the observations.

\section{June 1993 Event}

Figure 4a shows an expanded plot of Ulysses measurements of solar wind speed. proton density, and proton pressure for the June 1993 event, which was observed at $4.6 \mathrm{AU}$ and $\mathrm{S} 32.5^{\circ}$ and which was associated with a soft solar $\mathrm{X}$ ray event observed by Yohkoh on the west limb of the Sun on May 31 ,
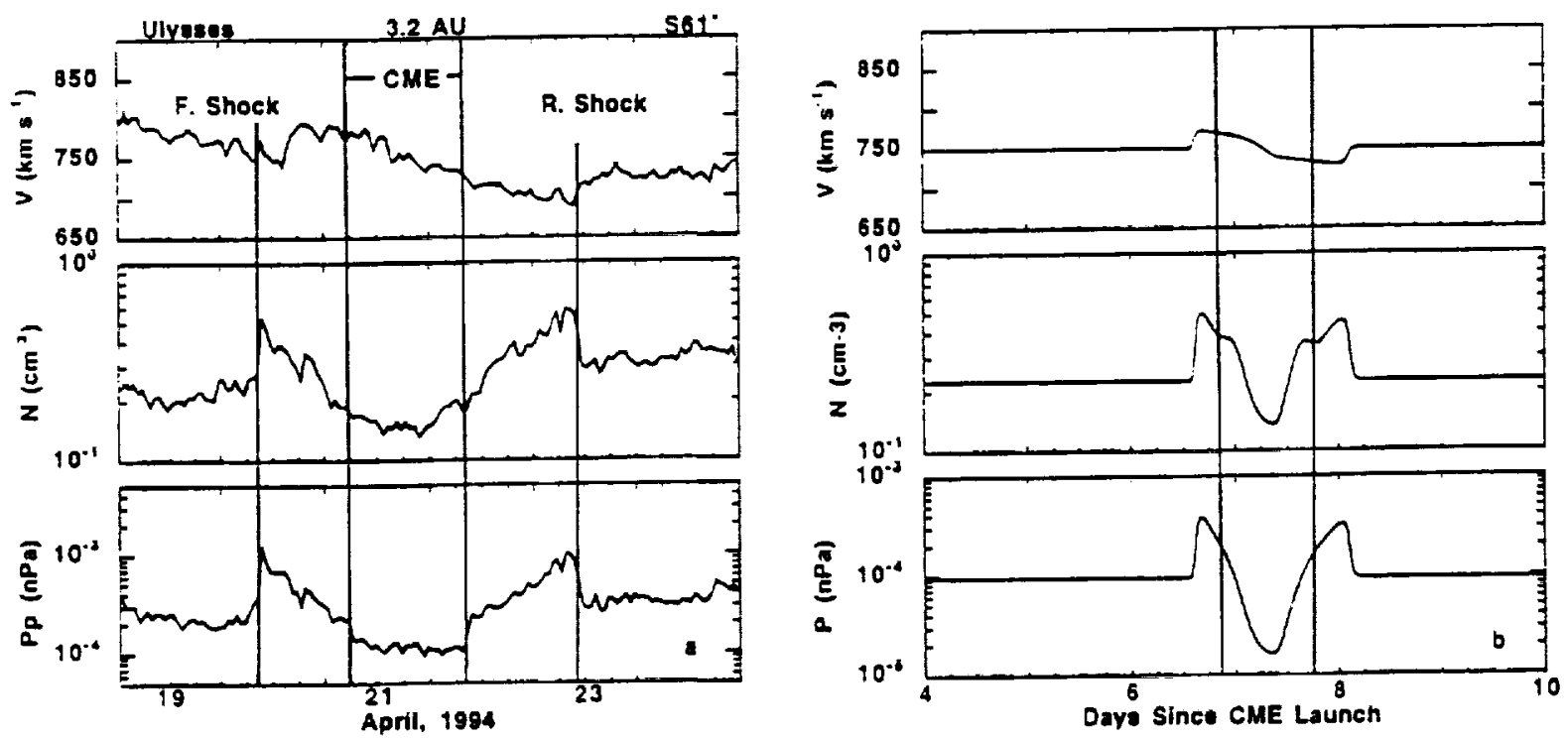

Figure 2. (a) Forward-reverse shock pair observed in the solar wind by Ulysses at $3.2 \mathrm{AU}$ and $\mathrm{S} 61^{\circ}$ in association with an overexpanded CME, bracketed by the solid vertical lines running from top to bottom of the plot. Parameters plotted are the solar wind speed. the proton number density, and the proton thermal pressure. (b) Simulated profile of the Ulysses event (see text and Figure 3). Vertical lines bracket the material introduced with a higher density at $0.14 \mathrm{AU}$ and thus identify the CME in the simulation. 

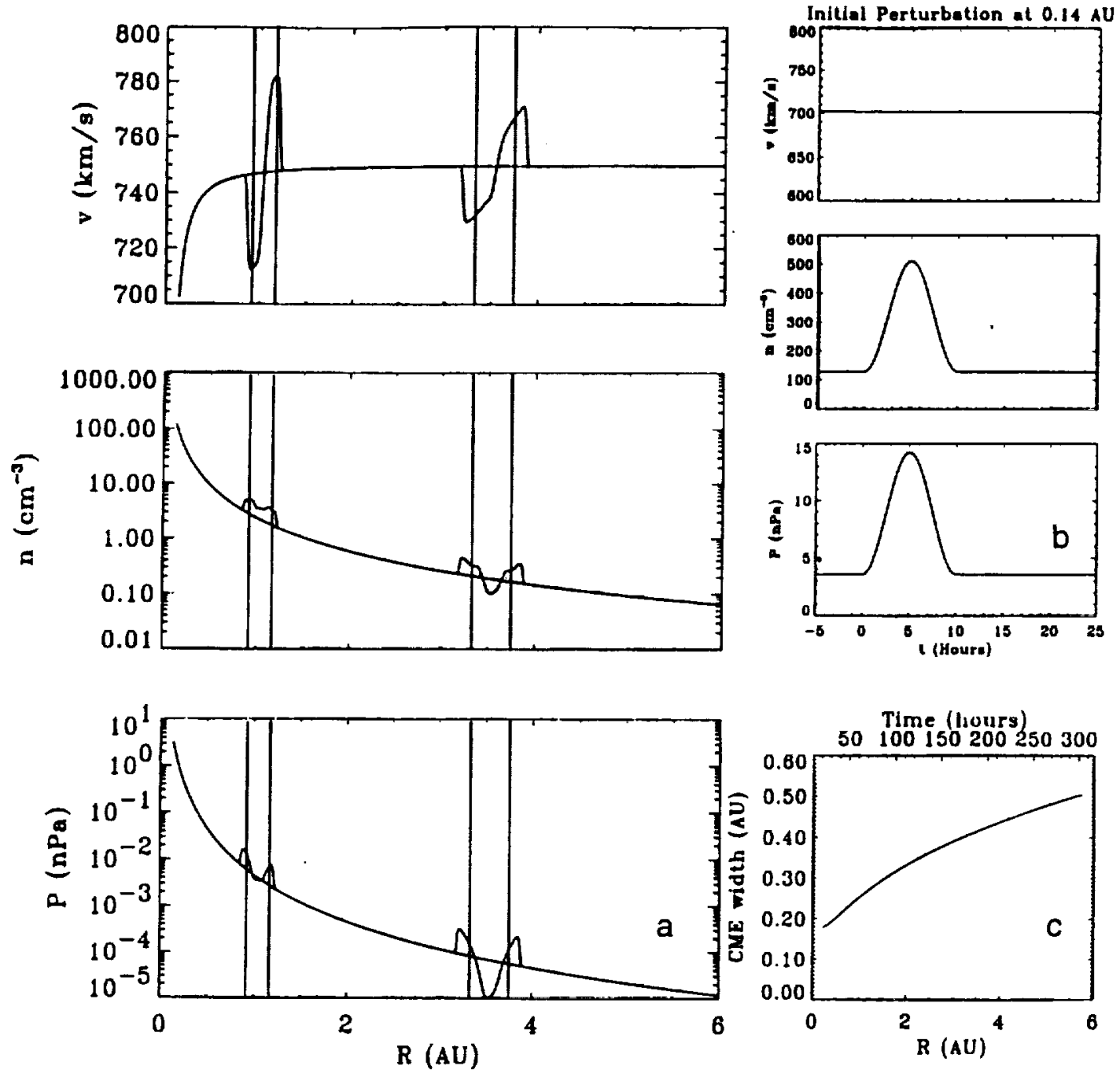

Figure 3 . A simulation of the Ulysses event shown in Figure 2a. (a) Solar wind speed, density, and pressure as functions of heliocentric distance 55 and 194 hours after initiation of the disturbance at 0.14 AU. Vertical lines bracket the material introduced with a higher density at $0.14 \mathrm{AU}$ and thus identify the CME in the simulation. (b) Initial conditions for the simulation at 0.14 AU. (c) Simulated CME width as a function of heliocentric distance.

1993 [Gosling et al., 1994a, 1995c]. Again, the CME material was identified in the plasma data by the presence of counterstreaming halo electrons. Magnetic field data reveal that this CME was also a "magnetic cloud" [e.g., Burlaga. 1991]. The speed of the center of the CME was $-740 \mathrm{~km} \mathrm{~s}^{-1}$, slower than that of the ambient wind ahead of the forward shock, and declined by $-180 \mathrm{~km} \mathrm{~s}^{-1}$ from the front to the rear of the CME. The trailing ambient wind had a speed approximately $125 \mathrm{~km} \mathrm{~s}^{-1}$ slower than that of the ambient wind ahead of the disturbance. As in the April 1994 event, minima in proton density and proton pressure occurred near the center of the CME, while maxima in those quantities occurred immediately downstream from the relatively weak shocks on either side of the CME. The reverse shock was offset from the edge of the CME more than was the forward shock. We calculate that the CME at Ulysses had a width of $-1.2 \mathrm{AU}$, while the total disturbance width from forward to reverse shock was $-2.0 \mathrm{AU}$.

Using our numerical code, we have simulated the June 1993 event by initiating a disturbance at 0.14 AUby increasing the density by a factor of four in a bell-shaped pulse 10-hours wide (similar to our previous example) while holding the temperature constant there, dropping the speed smoothly from 700 to $550 \mathrm{~km} \mathrm{~s}^{-1}$, and leaving it at $550 \mathrm{~km} \mathrm{~s}^{-1}$ thereafter. This mimics ejection from the Sun of a dense, high-pressure CME that is already expanding at the inner boundary of the calculation and whose central speed is less than that of the ambient wind ahead and greater than that of the ambient wind behind. Figure $4 \mathrm{~b}$ shows temporal profiles of the resulting disturbance (speed, density, and pressure) at $4.6 \mathrm{AU}$, while Figure 5 shows snapshots of the radial evolution of the disturbance 55 and 194 hours after initiation, the initial perturbation parameters at $0.14 \mathrm{AU}$, and $\mathrm{CME}$ width as a function of heliocentric distance.

This disturbance expands more rapidly than the disturbance in our previous example (Figures $2 b$ and 3 ) owing to the initial decline in speed across the CME. As a result, at any given heliocentric distance densities and pressures within the CME are lower than in the previous example and the CME width is greater. After an initial decrease in the rate of 

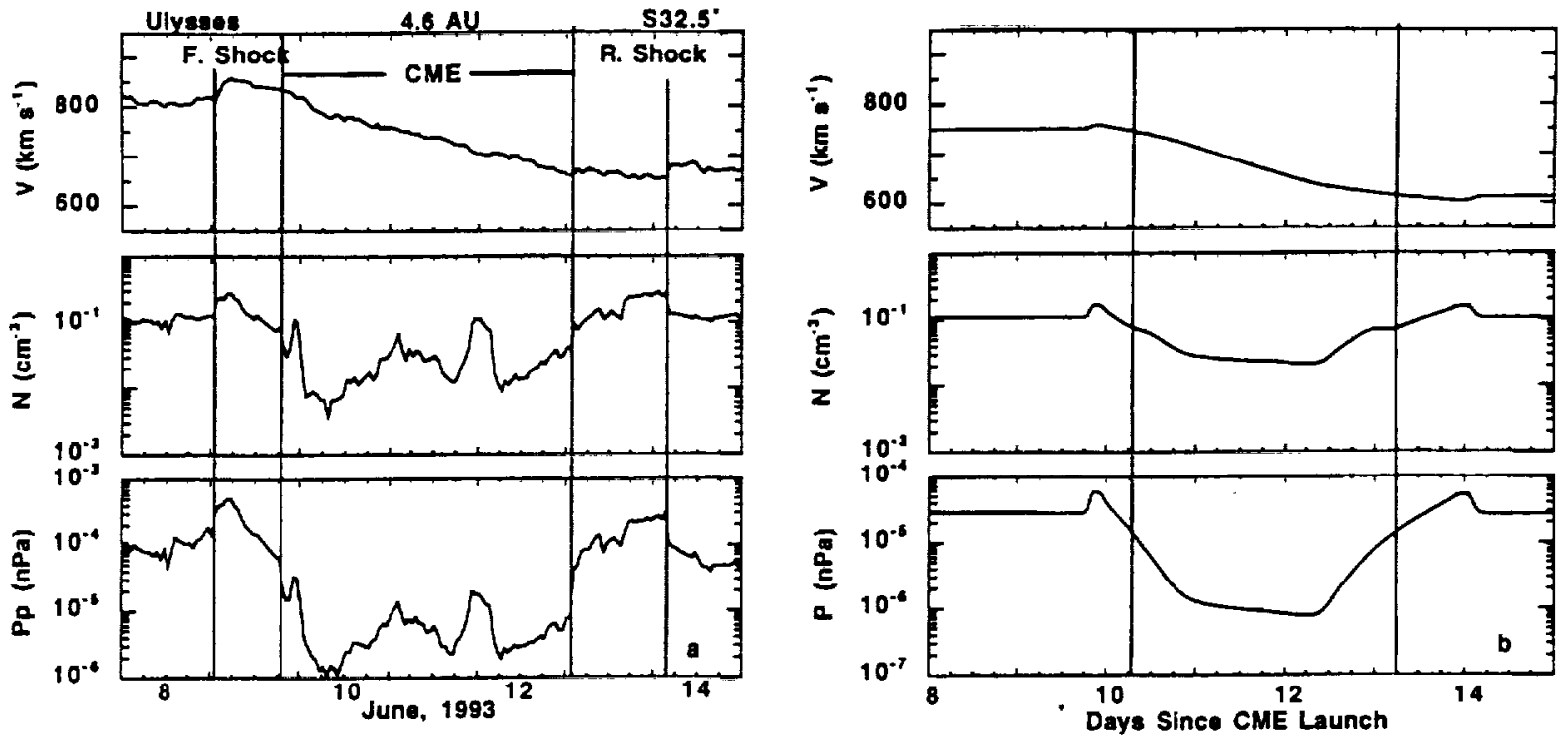

Figure 4. (a) Same as Figure 2a, but for an event observed by Ulysses at $4.6 \mathrm{AU}$ and S33 . (b) Simulated profile of the Ulysses event (see text and Figure 5).
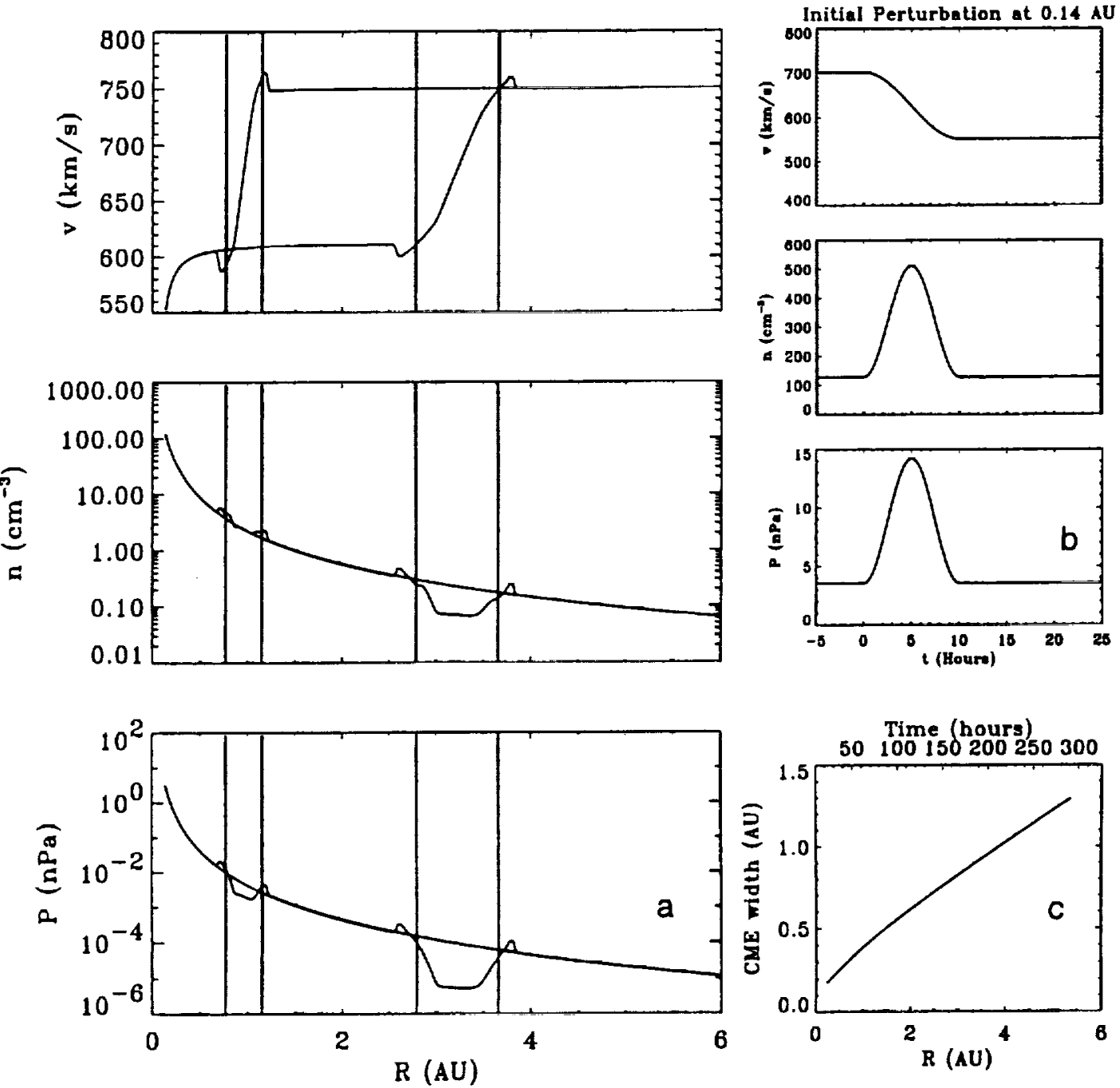

Figure 5. Same as Figure 3, except for a simulation of the Ulysses event shown in Figure 4a. 
expansion, owing to the CME's interaction with the surrounding ambient wind, the rate of expansion is nearly constant with increasing heliocentric distance. In the absence of the initial overpressure within the CME, the initial decline in speed across the CME would produce a rarefaction wave that would propagate both forward and backward into the ambient wind. As it propagates the wave would produce a deceleration of the leading wind and an acceleration of the trailing wind (see Gosling and Riley [1996] for a discussion of these effects). An initial high internal pressure within the CME produces the opposite effect: compressive waves that propagate both forward and backward into the ambient plasma and that accelerate the leading wind and decelerate the trailing wind. These waves, and the accelerations they produce, are weaker than in the previous example because of the competing effect of the rarefaction. Nevertheless, the waves do steepen into shock-like structures far from the Sun.

Comparison of Figures $4 a$ and $4 b$ reveals that the simulation has reproduced most of the essential aspects of the Ulysses observations of the June 1993 event including (1) the broad minima in density and pressure within the CME, (2) the declining speed gradient across the CME, (3) the weak forward and reverse waves bounding the disturbance with the pressure maximizing immediately downstream from the shocks. (4) the asymmetric placement of the forward and reverse shocks relative to the edges of the CME, and ( 5 ) the total width of the CME. The simulation does less well in reproducing the magnitudes of the density and pressure minima within the CME and, as in the comparison provided by Figure 2 , underestimates the offsets between the shocks and the edges of the CMEbecause the characteristic speed with which pressure disturbances propagate in the simulation is less than in the real solar wind. We emphasize that both the initial speed gradient and the initial overpressure within the CME are necessary ingredients in the simulation's ability to reproduce the essential characteristics of the observed event.

\section{August/September 1993 Event}

Figure 6a shows an expanded plot of Ulysses measurements of solar wind speed, proton density, and proton pressure for the last of the three events shown in Figure $I$ when the spacecraft was at a latitude of $\sim \mathrm{S} 38^{\circ}$ and $-4.4 \mathrm{AU}$ from the Sun. The CME was identified by the presence of counterstreaming (along the magnetic field) suprathermal electrons, occurred near the middle of a relatively large declining speed gradient, had a width of $-1.2 \mathrm{AU}$, was characterized by broad minima in density and pressure, and was followed by a relatively weak reverse shock that was propagating into the trailing solar wind. This reverse shock has previously been misidentified as being associated with a corotating interaction region (CIR) [Gosling et al.. 1993]. No forward shock or compressive wave was evident on the leading edge of the disturbance despite the presence of a reverse shock trailing the $\mathrm{CME}$ and despite the evidence that the CME was expanding.

We suspect that the lack of an observable forward wave ahead of the August/September 1993 event was a consequence of the fact that the wind ahead of the CME was running away from the CME faster than the CME could expand into it. Accordingly, we have attempted to simulate this event by initiating a disturbance at 0.14 AU by increasing the density of a factor of 4 in a bell-shaped pulse 20-hours wide while holding the temperature constant there, and by decreasing the speed from 700 to $550 \mathrm{~km} \mathrm{~s}^{-1}$ over a 10 -hour wide interval and maintaining the speed at $550 \mathrm{~km} \mathrm{~s}^{-1}$ thereafter. Note that although somewhat similar to the perturbation used in the previous simulation (Figures $4 b$ and 5 ), the density perturbation here is twice as broad (and hence the pressure gradients within the CME are only half as strong) and the central CME speed is $75 \mathrm{~km} \mathrm{~s}^{-1}$ lower. This mimics ejection from the Sun of a broad, dense, high-pressure CME whose leading portion is already expanding at the inner boundary of
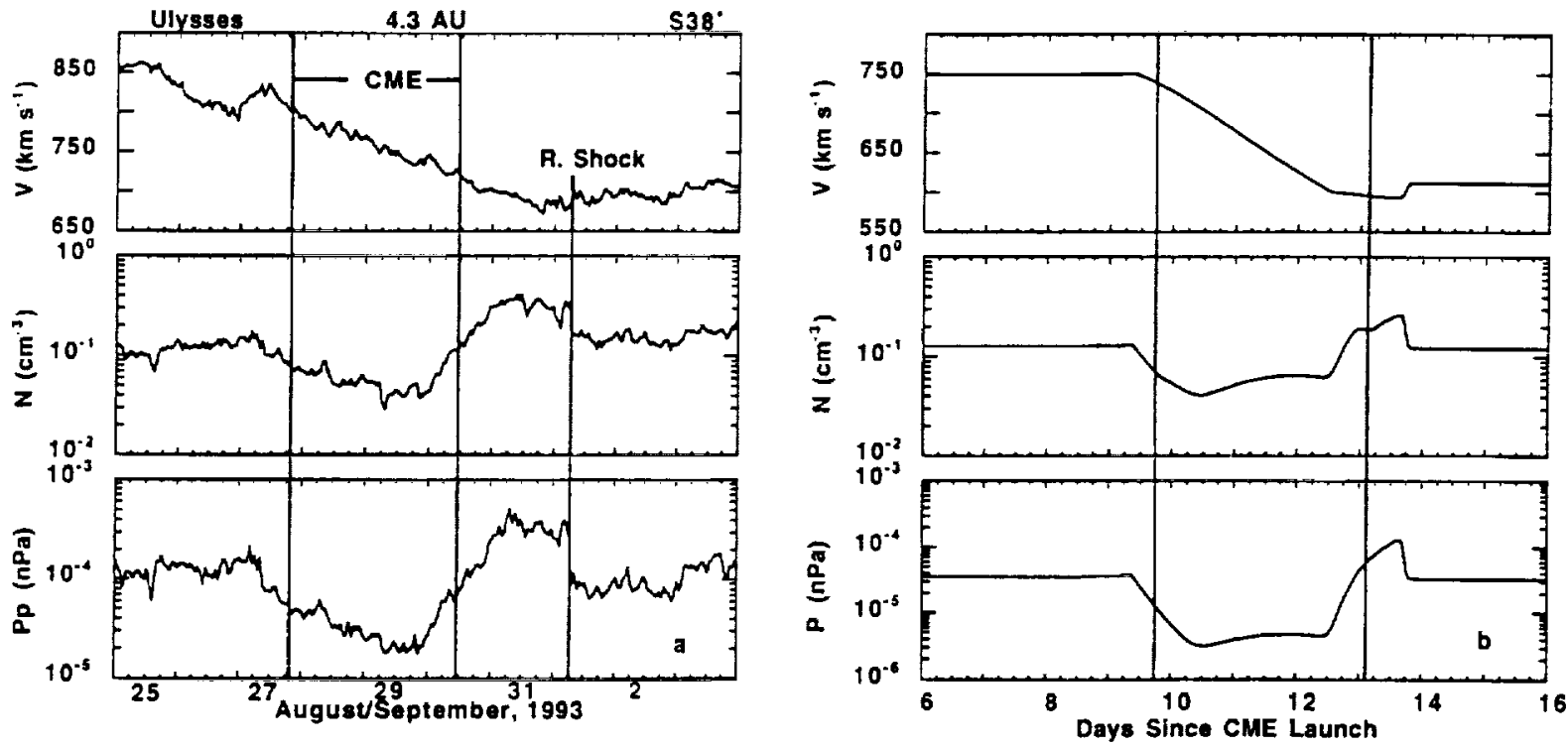

Figure 6. (a) Same as Figure 2a, but for an event observed by Ulysses at $4.3 \mathrm{AU}$ and S38 $8^{\circ}$ (b) Simulated profile of the Ulysses event (see text and Figure 7 ). 
the calculation and whose central speed is $150 \mathrm{~km} \mathrm{~s}^{-1}$ less than that of the ambient wind ahead but the same as that of the trailing wind. Figure $6 \mathrm{~b}$ shows the resulting disturbance temporal profiles at $4.3 \mathrm{AU}$, while Figure 7 shows snapshots of the radial evolution of the disturbance 55 and 194 hours after initiation, the initial perturbation parameters at 0.14 $A U$, and $C M E$ width as a function of heliocentric distance.

As in the previous simulation (Figures $4 b$ and 5), the CME in this simulation expands both because it has a high initial pressure and because its leading portion was already expanding when introduced at the inner boundary of the calculation. In this case though the disturbance develops in a more asymmetric fashion because of the asymmetry of the initial speed perturbation and the weaker pressure gradients associated with a broader initial disturbance. The forward wave associated with overexpansion (i.e., that portion of the expansion driven by the initial high internal pressure) of the CME is extremely weak in this case because the ambient wind ahead runs away from the CME faster than the CME can effectively expand into it. Nevertheless, the forward wave is barely visible in both Figures $6 \mathrm{~b}$ and 7 as a small positive bump in speed, density, and pressure running ahead of the CME; such a weak wave would be extremely difficult to detect in the observations. For the most part, however, the leading edge of the disturbance is dominated by the rarefaction associated with the initial declining speed gradient. Propagation of this rarefaction into the leading solar wind lowers the density, pressure, and speed of the wind immediately ahead of the CME. On the other hand, a reverse compression wave develops at the rear of the disturbance driven by the overexpansion of the rear portion of the CME into the trailing wind of the same speed. The trailing wind is slowed and compressed as it interacts with this wave. At large distances from the Sun the reverse wave eventually steepens into a shock.

Comparison of Figures $6 \mathrm{a}$ and $6 \mathrm{~b}$ indicates that our simulation has once again produced many of the essential features of the observed event including (1) the broad minima in density and pressure within the CME, (2) the declining speed gradient across the CME. (3) the rarefaction at the leading edge of the disturbance. (4) the relatively weak reverse shock and region of compression at the trailing edge of the disturbance, and (5) the magnitude of the density minimum within the CME. On the other hand, the simulated CME is somewhat broader than the observed CME and has a lower pressure minimum, and the reverse shock does not stand off as much from the rear of the CME as it does in the observations. Again, we emphasize that both the initial speed gradient and
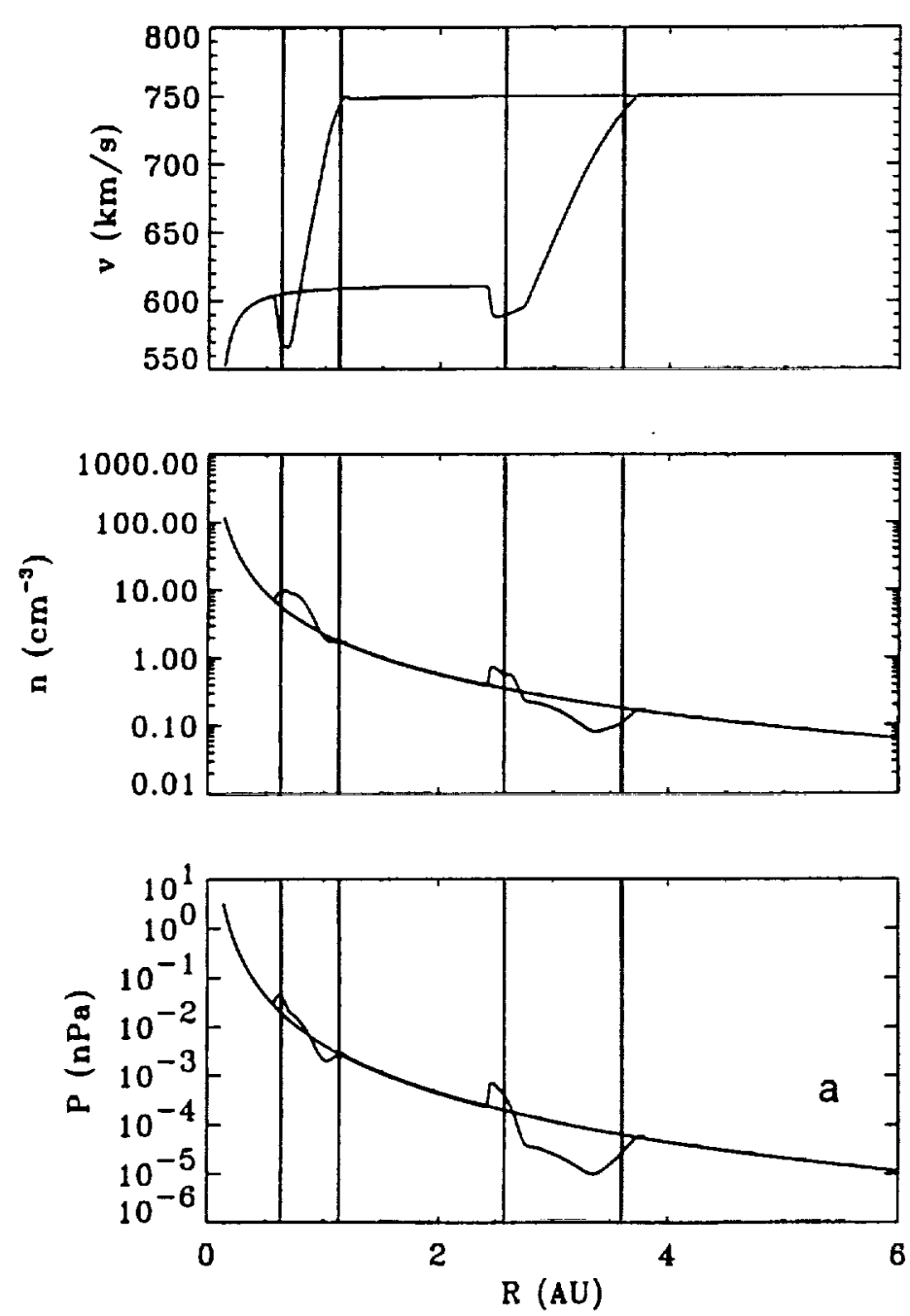
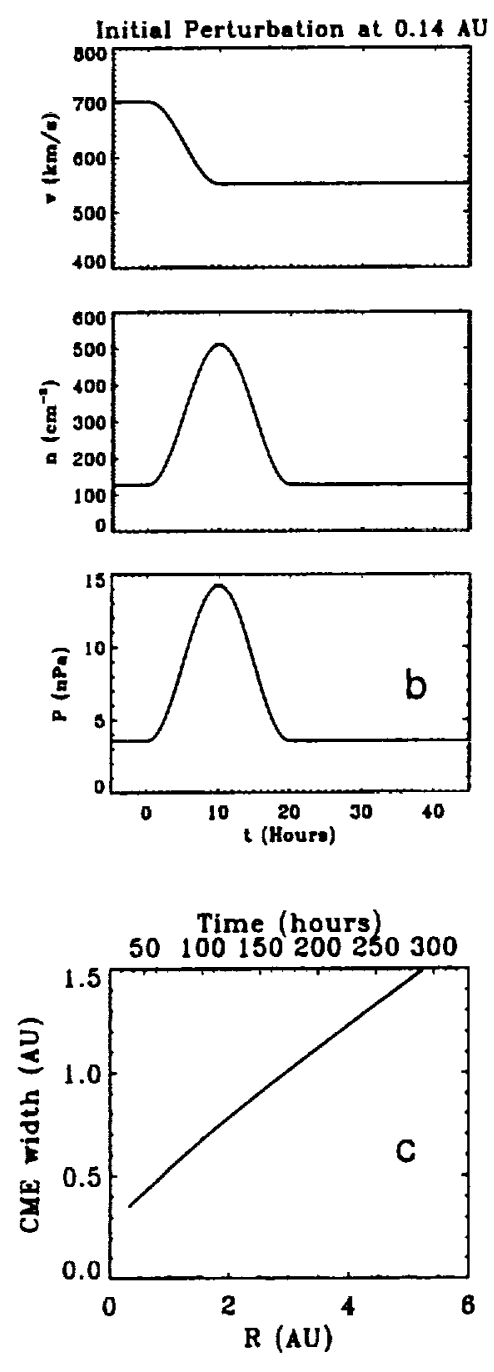

Figure 7. Same as Figure 3, except for a simulation of the Ulysses event shown in Figure 6a. 
the initial overpressure within the CME are necessary ingredients in the simulation's ability to reproduce the essential characteristics of the observed event.

\section{October 1996 Event}

A CME-driven disturbance was observed by Ulysses in October 1996 at the northern edge of the band of solar wind variability [Gosling es al., 1997]. Figure 8a shows measurements of solar wind speed, proton density, and proton pressure for this event, which was observed when Ulysses was at a heliocentric distance of $\sim 4.4 \mathrm{AU}$, at $\mathrm{N} 24^{\circ}$, and approximately $37^{\circ}$ behind the west limb of the Sun as observed from Earth. The solar event that produced this disturbance was detected by the coronagraph experiment on SOHO as a large and relatively fast CME originating behind the west limb of the Sun on October 5, 1996 (R. Howard and R. Schwenn, personal communication, 1996). The CME material was identified in the Ulysses measurements by prolonged intervals of counterstreaming suprathermal electrons, anomalously low proton temperatures, and abnormally high helium abundance [see Gosling et al.. 1997]. The CME had a radial width of $-2.5 \mathrm{AU}$ and was still expanding rapidly as it passed Ulysses. The CME was also characterized by abnormally low proton density and pressure (Figure 8a) and had a distinct magnetic signature (R. Forsyth, personal communication, 1996). It was preceded by a forward shock propagating into the high-speed wind ahead even though the bulk of the CME had a lower speed than that of the ambient wind ahead of the shock. No reverse shock was observed in association with this event; however, a second. relatively weak, forward shock was observed propagating through the back portion of the CME.

The observations suggest that the forward shock ahead of the CME was produced by overexpansion of the CME and that the relative motion between the CME and the slower, trailing solar wind was the reason why no reverse shock was observed. In order to attempt to reproduce this type of event we have initiated a disturbance at the inner boundary of our numerical code by increasing the density by a factor of 6 in a bell-shaped pulse 10-hours wide while simultaneously dropping the speed smoothly from 700 to $550 \mathrm{~km} \mathrm{~s}^{-1}$ and then allowing the speed to continue to drop to $400 \mathrm{~km} \mathrm{~s}^{-1}$ over the succeeding 10 hours. This mimics ejection from the Sun of a dense. highpressure CME that is already expanding at the inner boundary of the calculation and that has a central speed slightly less than that of the ambient solar wind ahead but considerably greater than that of the trailing solar wind. Figure $8 \mathrm{~b}$ shows the resulting disturbance temporal profiles at $4.5 \mathrm{AU}$. while Figure 9 shows snapshots of the radial evolution of the disturbance 55 and 194 hours after initiation. the initial perturbation parameters at $0.14 \mathrm{AU}$. and $\mathrm{CME}$ width as a function of heliocentric distance.

The disturbance in this simulation develops asymmetrically because of the asymmetry of the initial speed perturbation. A compressive wave, driven by the high initial internal pressure of the CME. propagates lorward into the fast ambient wind ahead of the CME and eventually steepens into a shock. This wave and the associated shock are weaker than they would be in the absence of the initial declining speed gradient because of the competing effect of the rarefaction produced by that speed gradient (see discussion relative to the simulation shown in Figures $4 \mathrm{~b}$ and 5). The reverse wave associated with overexpansion is considerably weaker than the forward wave because, in effect. the CME is running away from the trailing plasma faster than it can expand into it. Nevertheless, the wave can be discerned in Figure 9 as the small positive bump in speed. density, and pressure behind the CME propagating back toward the Sun in the CME rest frame. It would be very difficult to identify such a weak wave in the observations. The trailing portion of the disturbance is dominated by the rarefaction associated with the initial large negative speed gradient. As in the other simulations presented in this paper. the expansion of the CME with increasing heliocentric distance eventually produces abnormally low densities and pressures within the CME at large distances from the Sun.

We note that the simulation reproduces (1) the broad minima in density and pressure within the October 1996 event, (2) the
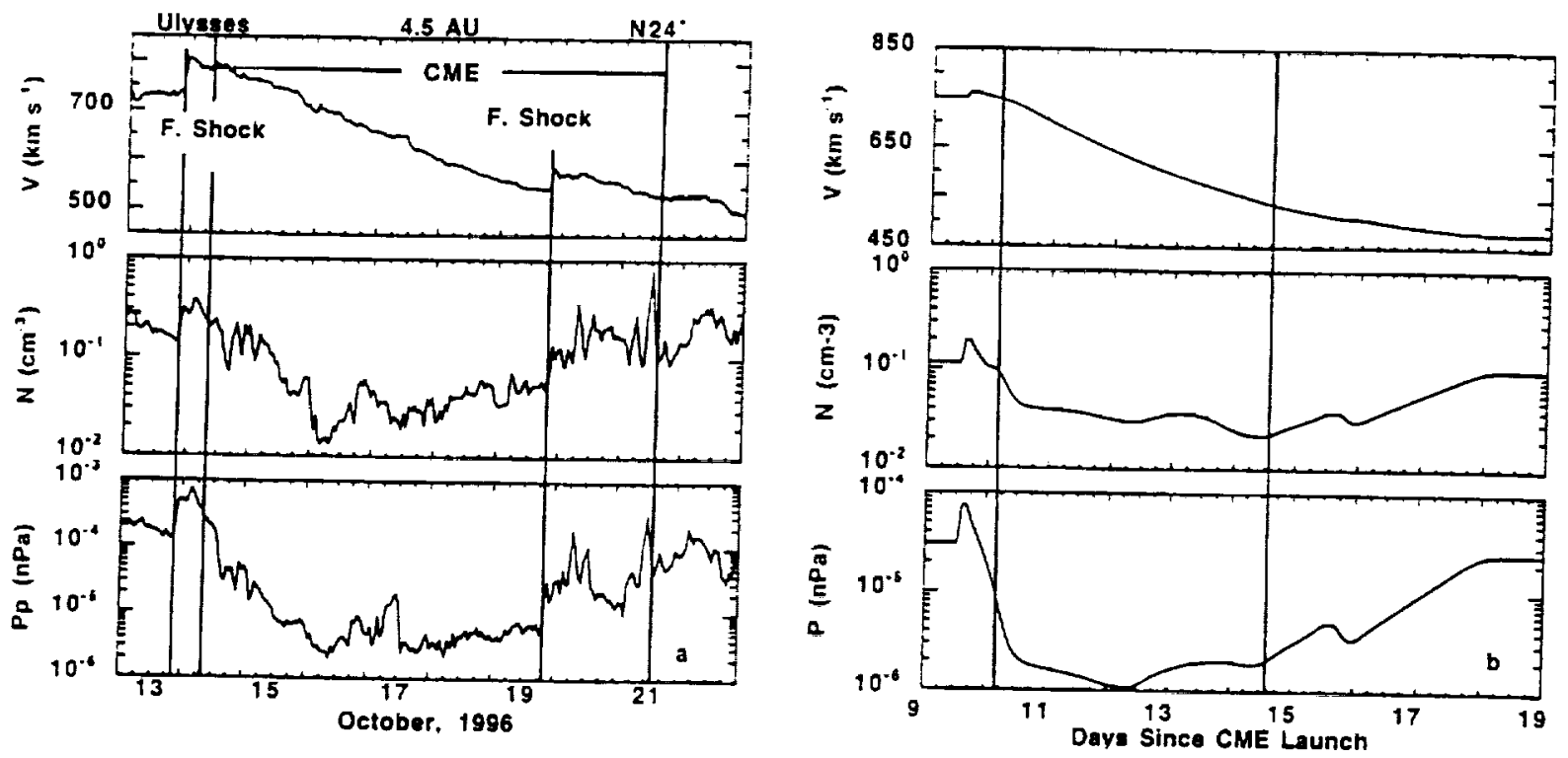

Figure 8. (a) Same as Figure 2a, but for an event observed by Ulysses at 4.5 AU and N24 $4^{\circ}$ (b) Simulated profile of the Ulysses event (see text and Figure 9). 

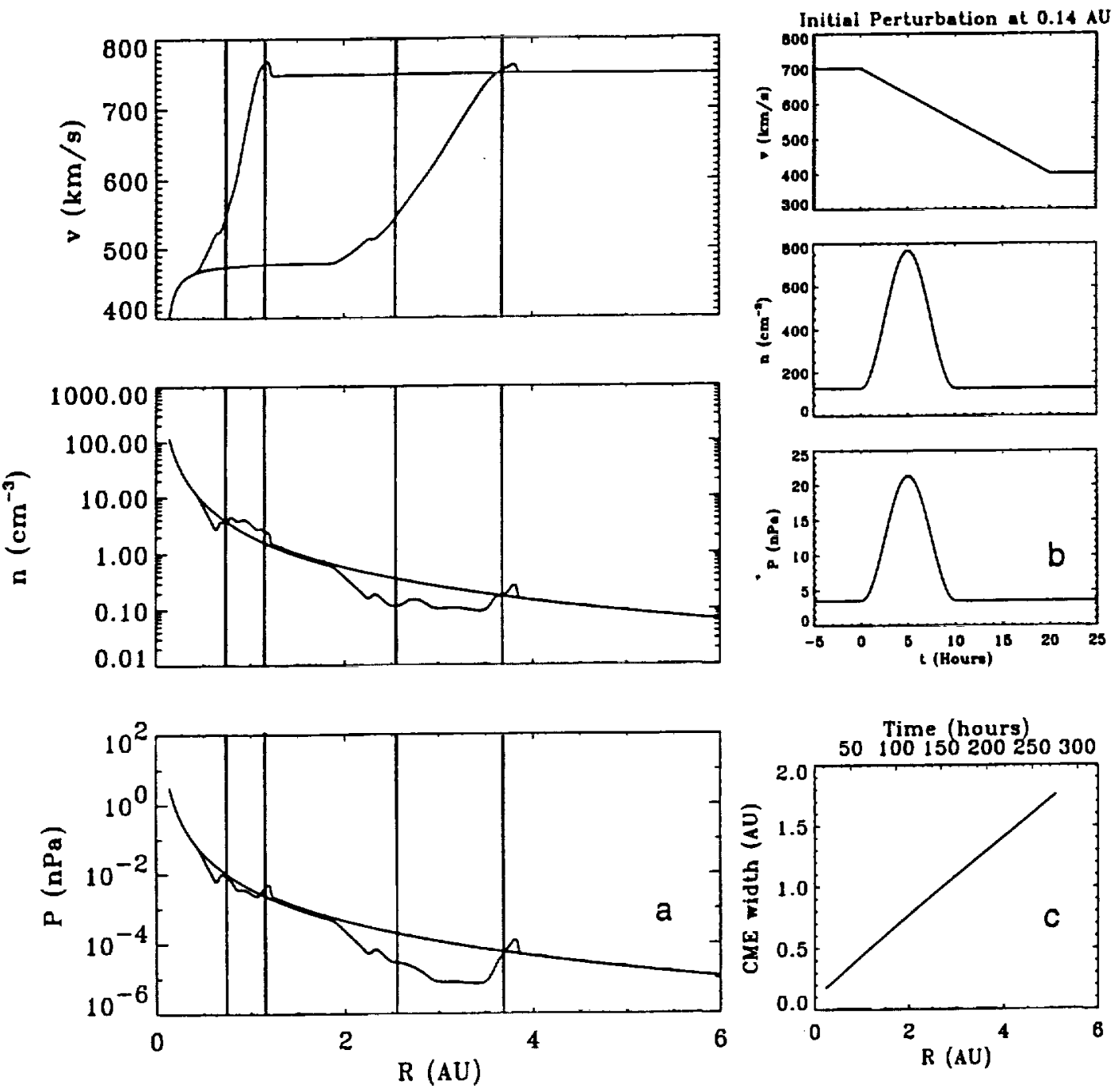

Figure 9. Same as Figure 3, except for a simulation of the Ulysses event shown in Figure 8a.

recovery in density and pressure at the rear of the disturbance, (3) the negative slope of the speed gradient across the event, (4) the forward shock propagating through the high-speed wind ahead of the CME. and (5) the offset between the shock and the leading edge of the CME. On the other hand, the observed shock was somewhat stronger than the shock produced in the simulation and the observed CME was -1 AU broader than obtained in the simulation. This simulation also does a reasonable job of reproducing the main characteristics of the February 1995 event observed at $1.4 \mathrm{AU}$. Again, we emphasize that both the initial speed gradient and the initial overpressure within the CME are necessary ingredients in the simulation's ability to reproduce the essential characteristics of the observed events.

\section{July 1993 Event}

Figure 10 shows an expanded plot of the second of the three events shown in Figure 1. The CME was identified using the combined plasma and magnetic field (not shown) data. It was both a counterstreaming suprathermal electron event and a "magnetic cloud" and was $-2.0 \mathrm{AU}$ wide when it passed the spacecraft. The front portion of the CME included deep minima in proton density and pressure associated with a long declining speed gradient, while the rear portion of the CME contained a rise in speed, density, and pressure terminating in a strong reverse shock propagating back into the trailing high-speed flow. (This reverse shock was previously thought to be associated with a CIR [Gosling et al., 1993; Tsurutaniet al. 1995], but it is now clear it is really associated with a high-speed flow overtaking a CME. Despite the earlier confusion in identification of this shock, our previous conclusion [Gosling et al., 1993, 1997] that CIRs in the opposite solar hemispheres have opposed north-south tilts as predicted by $P i z=$ [1991] remains unchanged.) There were no forward shocks associated with this event; however, the rise in speed, density, and pressure within the CME on July 24 was associated with a forward wave propagating through the back portion of the CME.

We have previously used this event to illustrate the processes by which a slow CME embedded in much faster leading and trailing ambient wind is accelerated up to high speed [Gosling and Riley, 1996]. Here we have attempted to simulate the event by initiating a disturbance at the inner boundary of our code by increasing the density by a factor of 4 and decreasing the speed by $400 \mathrm{~km} \mathrm{~s}^{-1}$ in simultaneous bellshaped pulses 40 -hours wide. This mimics the ejection of a very broad, dense, slow CME into the high-speed solar wind. 

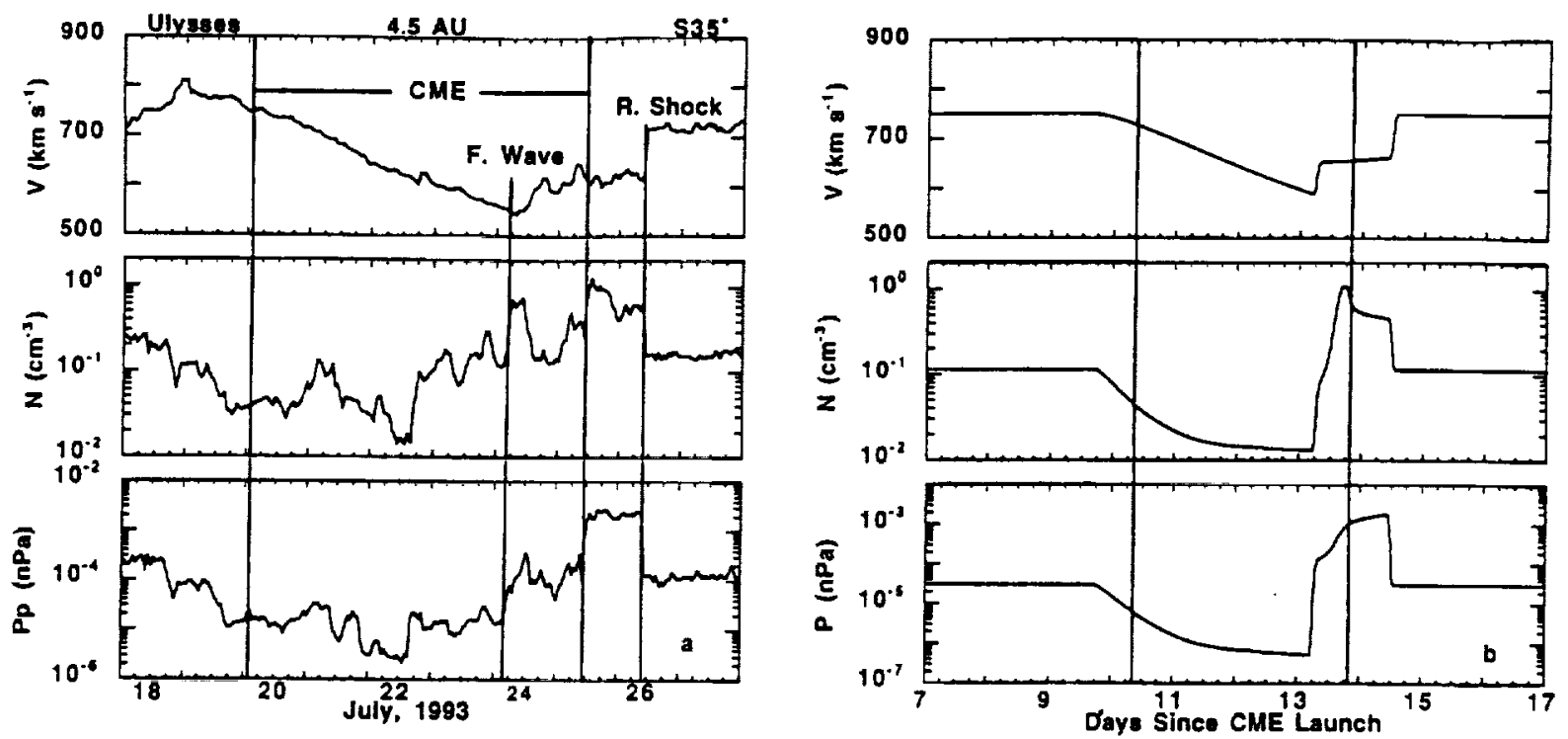

Figure 10 . (a) Same as Figure 2a, but for an event observed by Ulysses at $4.5 \mathrm{AU}$ and $\mathrm{S} 35^{\circ}$. Adapted from Gosling and Riley [1996] . (b) Simulated profile of the Ulysses event (see text and Figure 11).

Figure $10 \mathrm{~b}$ shows the resulting disturbance temporal profiles at 4.5 AU, while Figure 11 shows snapshots of the radial evolution of the disturbance 55 and 194 hours after initiation, the initial perturbation parameters at $0.14 \mathrm{AU}$, and $\mathrm{CME}$ width as a function of heliocentric distance.

A rarefaction develops in the leading portion of the disturbance that propagates both backward through the CME and forward into the ambient wind ahead. This rarefaction is a result of the declining speed gradient in the leading portion of the initial perturbation and is less pronounced near 1 AUthan it would be in the absence of an initial density/pressure perturbation. The forward propagation of this rarefaction produces a deceleration of the leading portion of the CME and the ambient wind ahead, while the backward propagation produces an acceleration of the remainder of the CME. At the same time, a region of strong compression forms on the trailing edge of the disturbance that, at large heliocentric distances, is bounded by a forward-reverse shock pair. This compression region is a result of the rising speed gradient in the trailing portion of the initial perturbation. The reverse wave propagates back into the trailing high-speed wind, compressing and decelerating it, while the forward wave propagates into the rear portion of the CME, compressing and accelerating it. One effect of the rarefaction and compression waves is to produce an overall acceleration of the CME with increasing distance from the Sun. As we have previously noted [Gosling and Riley, 1996], the effect of an initial density/pressure perturbation in events such as this is to broaden both the CME and the overall disturbance, to weaken the forward wave and retard its propagation into the CME. to strengthen the reverse shock behind the CME, and to lessen the overall acceleration of the CME. It is notable in this example that the forward and reverse waves associated with overexpansion of the CME are not evident in the simulation results. This is partially a consequence of the weaker pressure gradients associated with a very broad initial perturbation. In addition, however, the reverse wave associated with overexpansion is effectively obliterated as it interacts with the forward wave associated with the compression in the trailing portion of the disturbance, while the forward wave never really develops because the ambient wind ahead runs away from the CME faster than the CME can expand into it.

Comparison of Figures $10 \mathrm{a}$ and $10 \mathrm{~b}$ reveals that the simulation reproduces most of the features of the disturbance observed by Ulysses at $4.5 \mathrm{AU}$ including (1) the broad and deep minima in density and pressure and the declining speed gradient within the front portion of the CME and extending into the ambient wind ahead, (2) the forward wave, compression, and rising speed in the trailing portion of the CME. (3) the strong reverse shock and compression in the ambient wind behind the CME, and (4) the magnitude of the overall (negative) speed and density perturbations. We note, however, that the simulation has done less well in reproducing the width of the CME and the offsets of the forward and reverse waves from the back edge of the CME. Further, the simulation produces a forward shock within the CME where only a forward wave was observed. We suspect that this latter fact is a consequence of the very low beta of the CME, an observed feature that can not be extracted from a gasdynamic simulation. (We have previously incorrectly stated that this CME was a high beta event [Gosling and Riley, 1996].)

\section{Discussion}

Ulysses has provided the first direct measurements of CMEs in the solar wind at high solar latitudes. These measurements have revealed several aspects of high-latitude CMEs that were not anticipated prior to Ulysses' journey. These include the generally high speeds of CMEs in the high-latitude wind far from the Sun [Gosling et al, 1994b] and the fact that overexpansion (i.e., an expansion driven by an initial high internal pressure) of the CMEs commonly produces forward and/or reverse shocks that propagate into the surrounding ambient wind and deep rarefactions within the CMEs themselves. Of eight certain CME/disturbances observed either within the high-latitude wind or at the outer edges of the low-latitude band of solar wind variability, six had one or more bounding shocks associated with overexpansion (see 

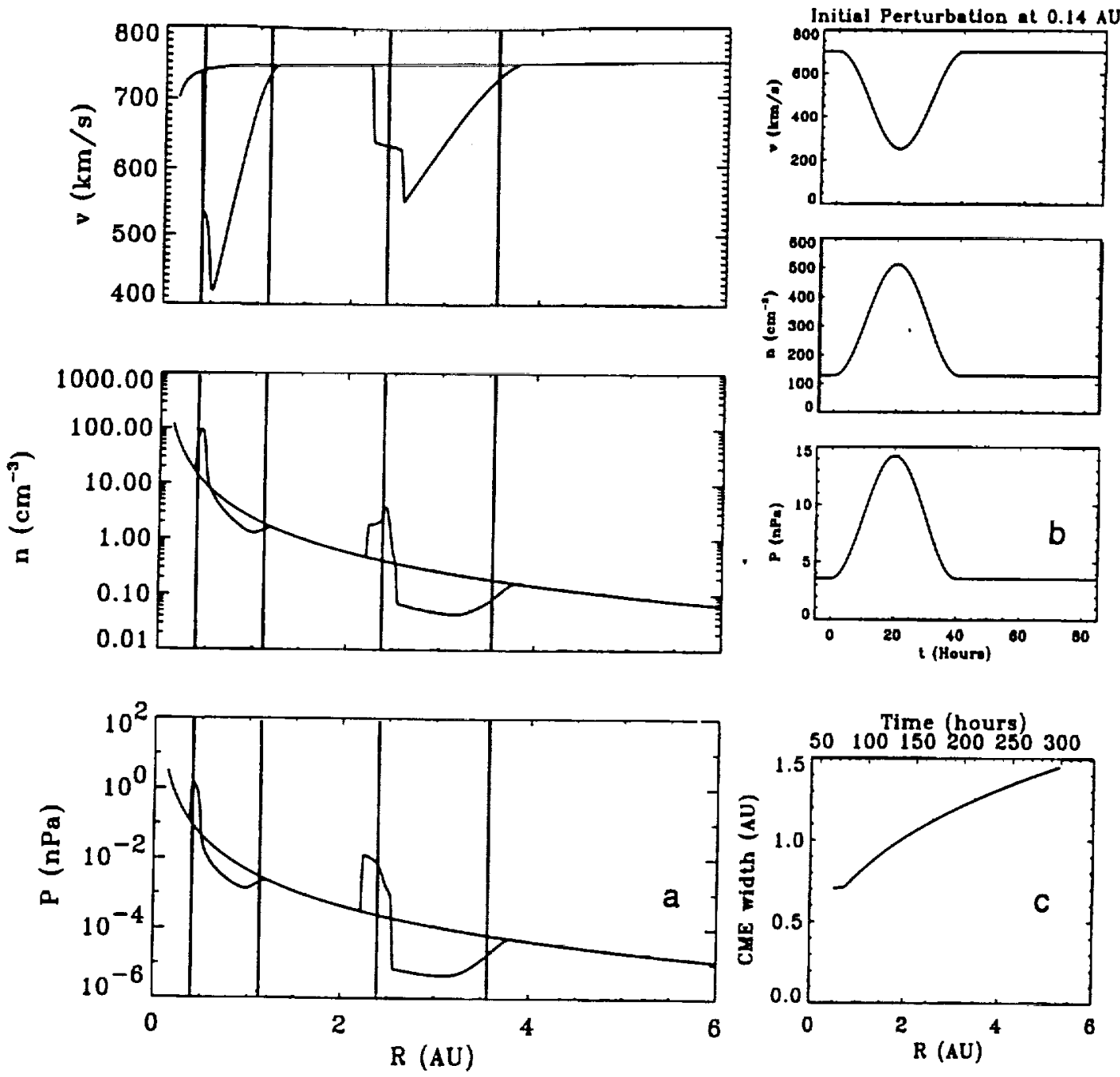

Figure 11. Same as Figure 3, except for a simulation of the Ulysses event shown in Figure 10a.

Table 1). In another event (July 1993) a fast solar wind flow overtaking the expanding CME produced a reverse shock but not a forward shock.

A basic theme of the present paper has been that $\mathrm{CME}$ overexpansion commonly occurs at high latitudes, but that the nature of the disturbance observed by a spacecraft far from the Sun depends importantly on the speed profile of the initial CME perturbation close to the Sun as well as the relative speed of the ambient wind ahead and behind. We have attempted to reproduce the main features of observed events using a simple one-dimensional, gasdynamic numerical code. Although we have used our experience with such calculations to choose initial perturbations close to the Sun that we believed would produce disturbances similar to what we have observed in the Ulysses data, we have not attempted to fine tune the simulations to reproduce all details of the observations. Indeed, we do not expect to be able to reproduce all aspects of these disturbances with a code that neglects the magnetic field and multidimensional effects. Moreover, our simulations have not been constrained by observations close to the Sun since coronagraph observations of the CMEs associated with the events observed by Ulysses are not available, with the exception of the most recent event observed in October 1996.
Experience indicates that real CMEs close to the Sun are far more complex than our simple simulations assume. Nevertheless, our simulations have produced disturbance profiles far from the Sun that are similar to those observed. We thus believe they provide a good starting point for understanding the dynamics and radial evolution of CMEs ejected into the high-speed solar wind at high heliographic latitudes.

Our combined observational and simulation results can be summarized as follows:

I. Most CMEs ejected into the high-latitude solar wind have a higher internal pressure than that of the surrounding ambient solar wind. This higher internal pressure is one reason why CMEs expand as they propagate out into the heliosphere.

2. When the ejection speed of a high-pressure CME is roughly the same as that of the ambient wind ahead and behind, overexpansion produces forward and reverse compressive wave that propagate into the ambient wind on either side of the CME and a rarefaction within the CME itself. At large distances from the Sun the compressive waves commonly steepen into relatively weak shocks and the CME continues to expand, forming an ever deeper rarefaction within the CME with increasing heliocentric distance. 
3. When a high-pressure CME is ejected into the wind with a greater leading edge speed than trailing edge speed, the initial declining speed gradient across the CME contributes substantially to the expansion of the CME and produces a broader disturbance far from the Sun than would be achieved by overexpansion alone. The expansion associated with a declining speed gradient does not produce compressive waves; rather, it produces a rarefaction that propagates forward and backward into the surrounding ambient wind. This rarefaction wave competes with and weakens the compressive waves associated with overexpansion.

4. When the ambient wind ahead of a high-pressure CME runs away from the CME faster than the CME can expand into it. no forward wave or shock is observed ahead of the CME. When a high-pressure CME runs away from slower trailing wind faster than it can expand into it, no reverse wave or shock is observed behind the CME. The rarefaction associated with such relative motion produces forces that contribute substantially to the overall expansion of the CME. These types of events, where relative motions produce rarefactions that effectively eliminate one or the other or the compressive waves associated with overexpansion and that enhance the expansion of the CMEs, tend to occur near the edge of the lowJatitude band of solar wind variability where the ambient wind speeds ahead and behind a CME are often quite different.

5. When a high-pressure CME is ejected into the wind with a speed that is considerably less than that of both the leading and trailing ambient wind. its evolution is dominated by the pressure gradients that develop in interplanetary space as a result of the relative motion. A strong rarefaction forms on the leading edge of the CME that propagates into the ambient wind ahead and causes the CME to expand. At the same time. a strong compression, bounded by forward and reverse waves. forms at the rear of the disturbance. The combined effect of the rarefaction and compression is to accelerate the CME to higher speed and decelerate the surrounding ambient wind. The simultaneous overexpansion of the CME modifies the above process. but the compressive waves associated with overexpansion are effectively obliterated by the compression and rarefaction associated with the motion of the slow CME relative to the faster surrounding ambient wind.

6. When a high-pressure CME has a speed considerably greater than that of the ambient wind, as often occurs at low heliographic latitudes. disturbance evolution is again dominated by the pressure gradients that develop in interplanetary space as a result of the relative motion between the CME and the ambient wind. In such cases. CME expansion is driven primarily by pressure gradients associated with the rarefaction formed as the CME pulls away from slower wind behind. the forward shock ahead of the CME is primarily a consequence of the relative motion rather than overexpansion. and the compressive waves associated with overexpansion either do not develop or are severely modified in transit from the Sun.

Acknowledgments. Work at Los Alamos was performed under the auspices of the US Deparment of Energy with suppor from an internal laboratory grant. Work at NOAA/SEC was supponed in part by NASA grants.

The Editor thanks S.W. Kahler and S. T. Suess for their assistance in evaluating this paper.

\section{References}

Alexander. D., H. S. Hudson. G. Slater, A. McAllister, and K. Harvey, The large scale coronal eruptive event of April 14. 1994. Solar Dynamic Phenomena and Solar Wind Consequences: Proceedings of the Third SOHO Workshop. Eur. Space A gency Spec. Publ. ESA SP. 373. 187. 1994.

Barne. S. J.. D. J. McComas, B. L. Barraclough. J. L. Phillips. K. J. Sofaly, J. C. Chavez. B. E. Goldstein, and R. K. Sakurai. The Ulysses solar wind plasma experiment. Astrom. Astrophys. Suppl. Ser., 92. 237. 1992.

Burlaga. L. F., Magnetic clouds. Plysics of the Inner Heliosphere 2, edited by R. Schwenn and E. Marsch. Springer-Verlag. New York, p 1,1991 .

Burlaga. L. F., E. Sittler. F. Mariani, and R. Schwenn. Magnetic loop behind an interplanetary shock: Voyager. Helios and IMP 8 observations. J. Geophys. Res.. 86.6673.1981.

Chen, J., Theory of prominence eruption and propagation: Interplanetary consequences. J. Geophys. Res.. 101. 27,499. 1996.

Gosling. J. T.. Transient phenomena in the solar atmosphere and solar wind, in Physics of Solar Planetary Environments. edited by D. J. Williams, p. 286, Washington. D. C.. 1976.

Gosling. J. T., Coronal mass. ejections and magnetic flux ropes in interplanetary space. in Physics of Magnetic Flux Ropes. Geophys. Monogr. Ser., vol. 58, edited by C. T. Russell. E. R. Priest. and L. C. Lee, p. 343, AGU, Washington, D. C., 1990.

Gosling. J. T., The solar flare myth, J. Geophys. Res., 98, 18.937. 1993.

Gosling. J. T., Magnetic topologies of coronal mass ejection events: Effects of 3-dimensional reconnection, in Solar Wind Eight, edited by D. Winterhalter, J. T. Gosling. S. R. Habbal. W. S. Kurth, and M. Neugebauer. p. 438, Am. Inst. of Phys., New York, 1996.

Gosling. J. T., and P. Riley. The acceleration of slow coronal mass ejections in the high-speed solar wind. Geophy.r. Res. Lett. 23. 2867. 1996.

Gosling. J. T., D. N. Baker, S. J. Bame, W. C. Feldman, R. D. Zwickl, and $E$. J. Smith. Bidirectional solar wind electron heat flux events, $J$. Geophys. Res.. 92. 8519. 1987.

Gosling. J. T.. S. J. Bame. D. J. McComas, J. L. Phillips, V. J. Pizzo, B. E. Goldstein. and M. Neugebauer, Latitudinal variation of solar wind corotating stream interaction regions: Ulysses, Geophyys. Res. Letr.. 20. 2789.1993

Gosling. J. T. S. J. Bame, D. J. McComas. J. L. Phillips. E. E. Scime. V. J. Pizzo. B. E. Goldstein, and A. Balogh. A forward-reverse shock pair in the solar wind driven by over expansion of a coronal mass ejection: Ulysses observations. Geophys. Res. Lert. 2J. 237, 1994a.

Gosling. J. T., S. J. Bame. D. J. McComas, J. L. Phillips. B. E. Goldstein, and $M$. Neugebauer. The speeds of coronal mass ejections in the solar wind at mid heliographic latitudes: Ulysses, Geophys. Res. Lett., 2I.1109. 1994b.

Gosling, J.T. D.J. McComas, J.L. Phillips, L.A. Weiss, V.J. Pizzo, B.E. Goldstein. and R.J. Forsyth. A new class of forward-reverse shock pairs in the solar wind, Geophys. Res. Letl, 2l, 227I, 1994c.

Gosling. J. T.. D. J. McComas, J. L. Phillips. V. J. Pizzo, B. E. Goldstein, R. J. Forsyth and R. P. Lepping. A CME-driven solar wind disturbance observed at both low and high heliographic latitudes. Geophys. Res. Lett. 22, 1753, 1995a.

Gosling. J. T.. S. J. Bame, W. C. Feldman. D. J. McComas. J. L. Phillips, B. E. Goldstein. M. Neugebauer, J. Burkepile, A. J. Hundhausen, and L. Acton. The band of solar wind variability at low heliographic latitudes near solar activity minimum: Plasma results from the Ulysses rapid latitude scan. Geophys. Res. Lett.. 22. 3329, 1995b.

Gosling. J. T., S. J. Bame. D. J. McComas, J.L. Phillips, A. Balogh, and K. Sirong. Coronal mass ejections at high heliographic latitudes: Ulysses, Space Sci. Rev., 72, 133, 1995c.

Gosling. J. T., S. J. Bame, W. C. Feldman, D. J. McComas. P. Riley, B. E. Goldstein. and $M$. Neugebauer. The northern edge of the band of solar wind variability: Ulysses at -4.5 AU. Geophys. Res. Letr., 24, $309,1997$.

Hundhausen. A. J.. Some macroscopic properties of shock waves in the heliosphere. in Collisionless Shocks in the Heliosphere: A Tutorial Review. Geophys. Monogr. Ser., vol. 34, edited by R. G. Stone and B. T. Tsurutani, p. 37. AGU. Washington. D. C.. 1985.

Hundhausen. A. J., The origin and propagation of coronal mass ejections, in Proceedings of the Sixth International Solar Wind Conference, edited by V. J. Pizzo, T. E. Holzer, and D. G. Sime. 
Tech. Note NCARTN-306+Proc, p. 181, Natl. Cent. for Atmos. Res., Boulder, Colo., 1988.

Hundhausen, A. J., A summary of SMM observations from 1980 and 1984-1989, in The Many Faces of the Sun, edited by K. Strong, J. Saba, and B. Haisch, Springer-Verlag, New York, in press, 1997.

Hundhausen, A. J., and R. A. Gentry, Numerical simulation of flaregenerated disturbances in the solar wind, J. Geophys. Res.. 74, 2908, 1969.

Kahler, S., Observations of coronal mass ejections near the Sun, in Proceedings of the Sixth International Solar Wind Conference, edited by V. J. Pizzo, T. E. Holzer, and D. G. Sime, Tech. Nore NCAR/N$306+$ Proc. p. 215, Natt. Cent. for Atmos. Res., Boulder, Colo.,1988.

Klein, L. W., and L. F. Burlaga, Magnetic clouds at 1 AU, J. Geophys. Res., 87, 613, 1982.

McAllister, A. H., M. Dryer, P. McIntosh, H. Singer, and L. Weiss, A Jarge polar crown coronal mass ejection and a "problem" geomagnetic storm: April 14-23, 1994. J. Geophys. Res., 101, 13,497, 1996.

Phillips, J. L., A. Balogh, S. J. Bame, B. E. Goldstein, J. T. Gosling. J. T., D. J. McComas, M. Neugebauer, N. R. Sheeley, and Y. -M. Wang, Ulysses at $50^{\circ}$ south: Constant immersion in the high-speed solar wind. Geophys. Res. Lett., 2I, I105, 1994.

Pizzo, V. J., Interplanetary shocks on the large scale: A retrospective on the last decade's theoretical efforts, in Collisionless Shocks in the Heliosphere: Reviews of Current Research, Geophys. Monogr. Ser., vol. 35, edited by B. T. Tsurutani and R. G. Stone, p. 51, AGU, Washington, D. C., 1985.

Pizzo, V. J., The evolution of corotating stream fronts near the ecliptic plane in the inner solar system, 2, Three-dimensional tilted-dipole fronts, J. Geophys. Res., 96, 5405, 1991.
Riley, P.. J. T. Gosling, and V. J. Pizzo, A two-dimensional simulation of the radial and latitudinal evolution of a solar wind disturbance driven by a fast, high-pressure coronal mass ejection, $J$. Geophys. Res., in

press, 1997.
Steinolfson, R. S., Theories of shock formation in the solar atmosphere, in Collisionless Shocks in the Heliosphere: Reviews of Current Research, Geophys. Monogr. Ser.. vol. 35, edited by B. T. Tsurutani and R. G. Stone, p. I, AGU, Washington, D. C., 1985.

Stone, J. M., and M. L. Norman, ZEUS-2D: A radiation magnetohydrodynamics code for astrophysical flows in two space dimensions, I. The hydrodynamic algorithms and tests, Astrophys. J.. $80,753,1992$

Tsurutani, B. T., C. M. Ho, J. K. Arballo, B. E. Goldstein, and A. Balogh, Large amplitude IMF fluctuations in corotating interaction regions: Ulysses at midlatitudes, Geophys. Res. Lett., 22, 3397, 1995.

Weiss, L. A., J. T. Gosling. A. McAllister, A. J. Hundhausen, J. T. Burkepile, J. L. Phillips, K. T. Strong, and R. J. Forsyth, A comparison of interplanetary coronal mass ejections at Ulysses with Yohkoh soft x-ray coronal events, Astron. Astrophys., 316. 384, 1996.

J. T. Gosling, D. J. McComas, and P. Riley, Los Alamos National Laboratory, Los Alamos, NM 87545. (email: jgosling@lanl.gov)

V. J. Pizzo, Space Environment Center, NOAA Boulder, CO 80303.

(Received February 14, 1997; revised April 15, 1997; accepted April 30, 1997.) 